\title{
Phaeochromocytoma:
}

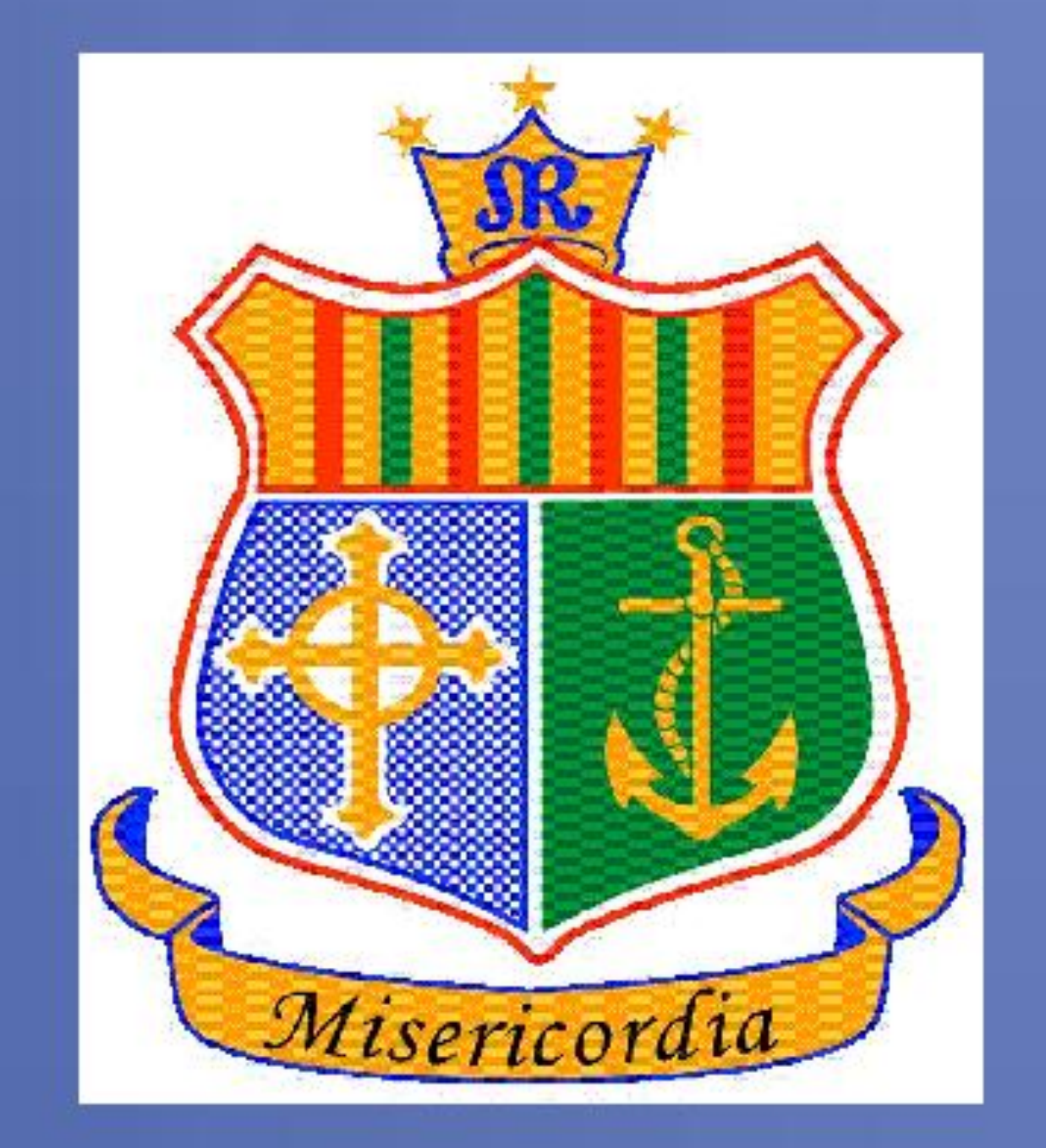

\section{The Mater Hospital experience over the past the past two decades}

\author{
B Monaghan 1, S Egan $_{2}$, G Mc Entee $_{3}$, MM Byrne $_{4}$
}

\section{1,2,4Department of Endocrinology Mater Misericordiae University Hospital, Dublin, Ireland \\ 3 Department of Surgery, Mater Misericordiae University Hospital, Dublin, Ireland}

\section{BACKGROUND}

Phaeochromocytoma is a rare neuroendocrine tumour. Incidence has been quoted at two to eight cases per million people. Over the past 18years, there were 22 cases of surgically resected phaeochromocytoma in the Mater Hospital.

\section{METHODS}

This is a retrospective study reviewing all cases of surgically resected phaeochromocytoma in a large tertiary referral centre between 1996 and 2014. There was with particular focus on preoperative care.

\section{RESULTS}

There were 22 cases of surgically resected phaeochromocytoma. $13.6 \%(n=3)$ of patients had malignant phaeochromocytoma.

\begin{tabular}{|lll|}
\hline FIG. 1:PATIENT DEMOGRAPHIC & \\
\hline Age at diagnosis: & $27-65$ yrs & Average 47yrs \\
\hline Gender: & Female & $63.6 \% \quad(n=14)$ \\
& Male & $36.4 \% \quad(n=8)$ \\
\hline Diagnosis: & Symptomatic & $81.8 \% \quad(n=18)$ \\
& Incidental & $18.2 \% \quad(n=4)$ \\
\hline Catecholamine & Hypertensive crisis & $14 \% \quad(n=3)$ \\
induced & Hypertensive retinopathy & $4.5 \% \quad(n=1)$ \\
complications at & Cardiomyopathy & $4.5 \% \quad(n=1)$ \\
presentation & Psychiatric disturbance & $4.5 \% \quad(n=1)$ \\
& Intra op -cardiac arrest & $4.5 \% \quad(n=1)$ \\
& & \\
\hline Concurrent medical \\
conditions at & Hypothyroidism & $18 \% \quad(n=4)$ \\
diagnosis & Type 2 Diabetes Mellitus & $18 \% \quad(n=4)$ \\
& Neurofibromatosis Type 1 & $9.0 \% \quad(n=2)$ \\
& Pregnancy 29/40 gestation & $4.5 \% \quad(n=1)$ \\
& Stage IV Colorectal Cancer & $4.5 \% \quad(n=1)$ \\
& Active cocaine use & $4.5 \% \quad(n=1)$ \\
& Type 1 Diabetes Mellitus & $4.5 \% \quad(n=1)$ \\
\hline
\end{tabular}

In terms of pre-operative optimization, medical treatment involves alpha blockade initially +/- beta blockade if tachycardic. It took $9.75+/-8.2$ days on average to obtain adequate alpha blockade. The mean dose of phenoxybenzamine used was $91.1+/-71.7 \mathrm{mg}$.

\begin{tabular}{l|l|l|l|}
\hline & Admission & Pre-operative & Post-operative \\
\hline $\mathrm{BP}(\mathrm{mm} / \mathrm{hg})$ & $152 / 92+/-34 / 18$ & $122 / 71+/-19 / 12$ & $108 / 63+/-15 / 10$ \\
$\mathrm{HR}(\mathrm{bpm})$ & $87+/-14$ & $74+/-16$ & $75+/-14$
\end{tabular}

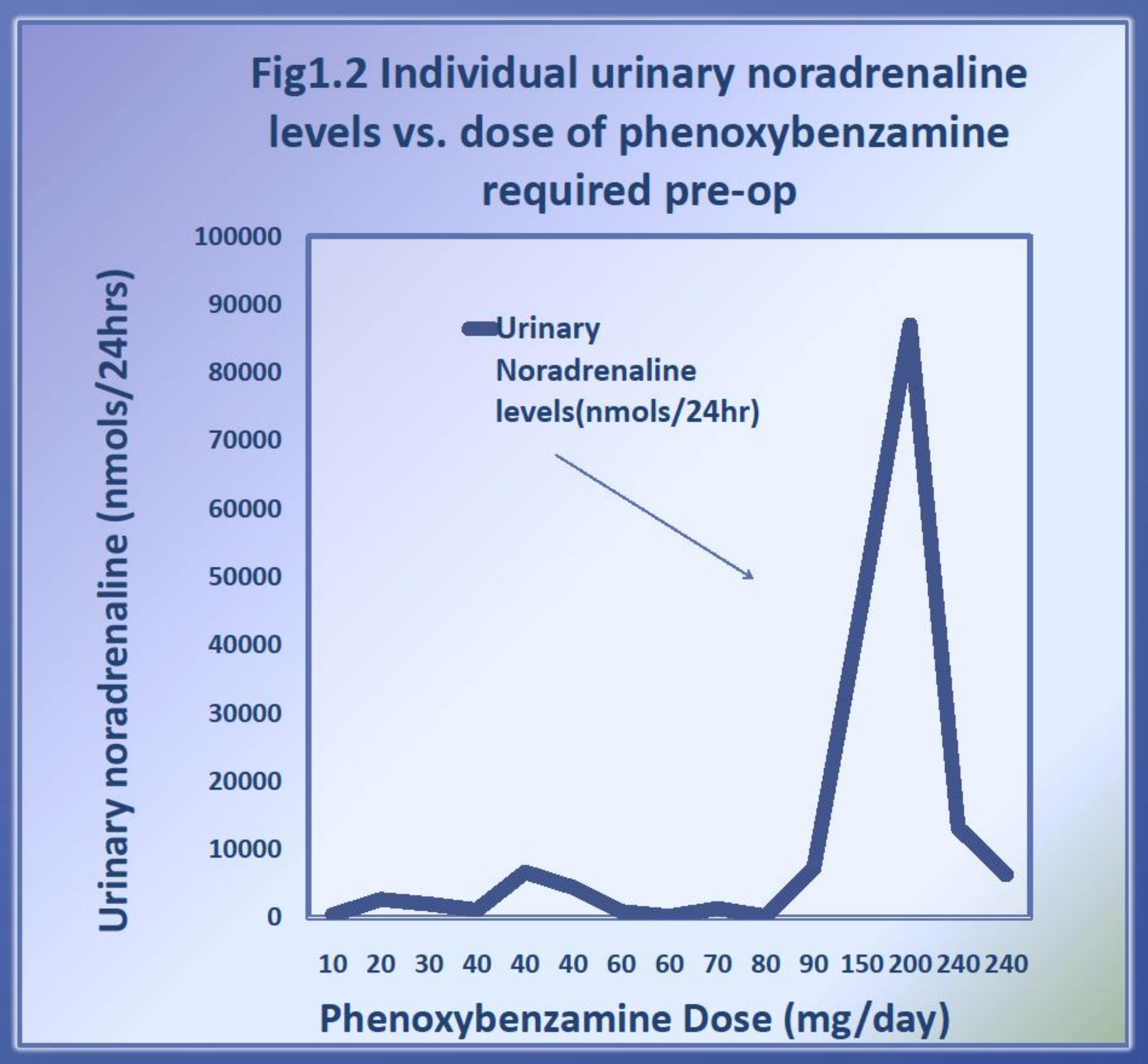

\begin{tabular}{|l|l|}
\hline FIG.2: PERIOPERATIVE OUTCOME & \\
\hline Surgical Approach & Laparotomy \\
\hline Unilateral Adrenalectomy & $95.5 \%(\mathrm{n}=21)$ \\
\hline Bilateral Adrenalectomy & $4.5 \%(\mathrm{n}=1)$ \\
\hline ICU/HDU Admission post-op & $81.8 \%(\mathrm{n}=18)$ \\
\hline Average ICU/HDU LOS (days) & $3.8(+/-1.6)$ \\
\hline Patients requiring inotropic support & $50 \%(\mathrm{n}=11)$ \\
\hline Inotropic support duration (days) & $2.9 \quad(+/-1.1)$ \\
\hline Immediate operative complications: & \\
\hline $\begin{array}{l}\text { Cardiac arrest at induction } \\
\text { Hypotension }\end{array}$ & $4.5 \%(\mathrm{n}=1)$ \\
\hline Hypertension & $50 \%(\mathrm{n}=11)$ \\
\hline Early post op complications: & $4.5 \%(\mathrm{n}=1)$ \\
\hline Lower respiratory tract infection & $4.5 \%(\mathrm{n}=1)$ \\
Renal haemorrhage & $4.5 \%(\mathrm{n}=1)$ \\
\hline Late post op complications & $\mathrm{Nil}$ \\
\hline Mortality related to surgery & $\mathrm{Nil}$ \\
\hline
\end{tabular}

\section{CONCLUSION:}

Over the past eighteen years, there has been twenty two successful surgical resections of phaeochromocytomas in the Mater Hospital. Each one presents a unique set of management challenges. Our report demonstrated that once diagnosed, intensive medical stabilization and specialised surgical expertise are required to prevent adverse outcomes perioperatively. 